\title{
Functional and Radiological Outcome in Delayed Presenting Closed Displaced Lateral Condyle Fracture of Humerus in Children
}

\author{
Afzal Hussain $^{1}$, Tariq Siddique ${ }^{1}$ and Syed Faraz UI Hassan Shah Gillani ${ }^{2}$ \\ ${ }^{1}$ Department of Orthopedic Surgery, Pakistan Society for Rehabilitation of Disabled, Lahore, Pakistan \\ ${ }^{2}$ Department of Orthopedic Surgery, King Edward Medical University / Mayo Hospital, Lahore, Pakistan
}

\begin{abstract}
This prospective cohort study was conducted from July 2013 to May 2019. The functional and radiological outcome in displaced, neglected fracture of lateral condyle of the humerus treated with open reduction and internal fixation (ORIF) with Kirschner wire was assessed. The sample size was 44 children aged between 2 to 15 years Milch type I and II displaced lateral condyle of humerus fracture, four weeks and older were included. Carrying angle and infection was assessed clinically. Union and elbow functions were evaluated radiologically using the Mayo elbow score. The mean age of the children was $6.82 \pm 2.83$ years. Fracture was Milch type I in 11 (25\%), and type II in 33 (75\%). Pre- and post-operatively, Mayo elbow score was $3.63 \pm 0.57$ and $1.56 \pm 0.50$, respectively. Good to excellent functional outcome, and union was observed in delayed presenting lateral condyle of humerus fractures.
\end{abstract}

Key Words: Lateral condyle humerus, Children, Delayed presentation, Mayo elbow score.

How to cite this article: Hussain A, Siddique T. Functional and Radiological Outcome in Delayed Presenting Closed Displaced Lateral Condyle Fracture of Humerus in Children. J Coll Physicians Surg Pak 2021; 31(01):107-109.

A fracture involving joints can be a disaster, if left untreated. Fracture lateral condyle of the humerus accounts for approximately 13 to 18 percent of the elbow joint. They commonly occur in children, at the peak age of 6 to 7 years. Untreated fractures of lateral condyle have a high risk of complications included, malunion, nonunion, avascular necrosis of ossific nucleus, ulnarnerve palsy, and angular deformity. ${ }^{1}$

These fractures are treated with different methods, according to the degree of displacement. It is generally accepted that lateral condyle fracture of the humerus with more than $2 \mathrm{~mm}$ displacement requires anatomical reduction. ${ }^{2}$

There is no consensus about the superiority of the surgical approach, the implant of choice, the postoperative period of immobilisation, and the management of neglected or late presenting fractures. ${ }^{3}$ In late presenting fracture, there are different treatment methods included internal fixation, correction of deformity secondary to fracture with osteotomy, ulnar nerve transposition, and sometimes combined procedures are required. ${ }^{4}$

Correspondence to: Dr. Tariq Siddique, Department of Orthopedic Surgery, Pakistan Society for Rehabilitation of Disabled, Lahore, Pakistan

E-mail: drtariqsiddiq@gmail.com

Received: June 21, 2017; Revised: March 19, 2020;

Accepted: March 30, 2020

DOI: https://doi.org/10.29271/jcpsp.2021.01.107
In children, there is the risk of cubitus valgus as the child grows older; and it is argued that osteosynthesis should be attempted early to prevent deformity and enable the condyle to participate in lowerhumerus growth. ${ }^{5}$

This will aid in producing evidence supporting open reduction and internal fixation of older displaced later condyle fracture in our setup. In this study, four weeks, and older displaced lateral condyle fracture humerus were treated with ORIF with K-wires; and the outcome was prospectively evaluated.

The study was conducted using a non-probability consecutive sampling technique at the Department of Orthopedic Surgery, Pakistan Society for Rehabilitation of Disabled, Lahore, from July 2013 to May 2019. Sample size of 44 children four week or older displaced lateral condyle humerus Milch type I \& II fractures were included; and children with a history of previous surgery were excluded.

After approval from the Institutional Review Board of the Hospital and, informed written consents from children and their parents, where required, were obtained. All children were managed with open reduction and internal fixation (ORIF) with Kirschner wire (K-wire) with bone grafting using Kocher approached (Figure 1). K-wires between 1.8-mm to 2.5-mm size were used. The site of bone graft was the metaphyseal area of the lateral lower condyle of the humerus or proximal ulna of the children. Carrying angle, infection, fracture union, and elbow function were evaluated. 
Table I: Paired t-test of mayo elbow score before and after the surgery.

\begin{tabular}{|l|c|c|c|c|}
\hline Variables & $\mathbf{n}$ & Mean & $\begin{array}{c}\text { Standard } \\
\text { deviation }\end{array}$ & p-value \\
\hline Pre-op mayo elbow score & 44 & 3.636 & 0.5742 & $<0.001$ \\
\hline Post-op mayo elbow score & 44 & 1.568 & 0.501 & \\
\hline
\end{tabular}

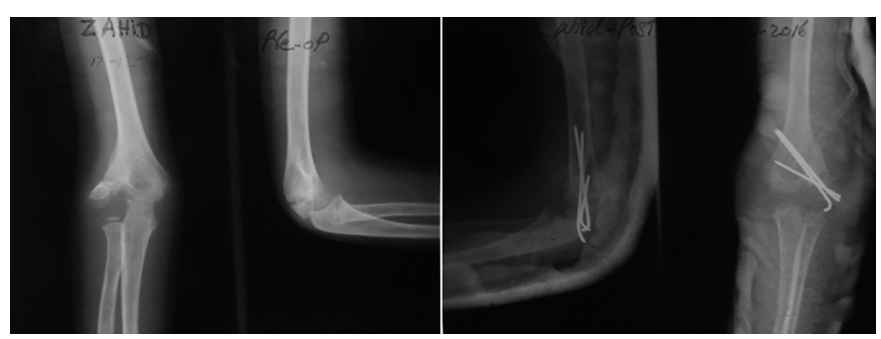

Figure 1: The pre- and postoperative radiographs of 12-year child with five-week older displaced later condyle fracture, treated with ORIF with K-wire with bone graft.

Children at $2^{\text {nd }}$ week, $6^{\text {th }}$ weeks, $12^{\text {th }}$ week, $24^{\text {th }}$ week, and $36^{\text {th }}$ week after the surgery were followed up. Carrying angle and infection was assessed clinically. Union and elbow functions were evaluated radiologically and using Mayo elbow score, respectively.

The wires were retained for a minimum of six weeks, and the limb protected in above elbow plaster of paris cast till radiological fracture union. Superficial infection was managed with debridement and culture sensitivity antibiotics. Deep infection was controlled following standard protocols.

Data was entered and analysed using SPSS version 20.0. The qualitative variables, like gender and side of the limb, was presented in frequencies and percentages. Quantitative variables including age, carrying angle, Mayo elbow score were presented as mean and standard deviations. Paired t-test was applied as a test of significance, and $p$-value $<5$ was taken as significant.

Out of a total of 44 children, $27(61.4 \%)$ were boys, and 17 (38.6\%) were girls. The age ranged from 2 to 13 years with mean of $6.82 \pm 2.83$ years. Majority $(28,63.6 \%)$ had already visited bone-setter, seven (15.9\%) did not visit for any treatment, while nine $(20.5 \%)$ children had displacement in plaster. The mean duration since injury was $4.93 \pm 0.76$ weeks. Milch type I fracture was seen in 11 (25\%), and type II was $33(75 \%)$. Superficial infection was noted in seven (15.9\%), and deep Infection was present in three $(06 \%)$ children. The mean pre- and postoperative carrying angle was $11.61 \pm 1.81$ and $9.34 \pm 0.83$, respectively. Union was observed in all children till the last follow-up. Preoperatively, the Mayo elbow score was fair in $12(27.3 \%)$ and poor in $72.7 \%$ with the mean score was $3.63 \pm 0.57$, and postoperatively it was improved to excellent in $28(63.6 \%)$ and good in $16(36.4 \%)$ with mean score $1.56 \pm 0.50$.

A paired t-test was applied, and the correlation of treatment was tested in terms of the Mayo elbow score before, and after the treatment, it was found significant $(p<0.001$, Talel).

Observers have variedly defined the 'delayed,' 'neglected' or 'late presentation' lateral condylar fractures (>3-6 weeks) in children, ${ }^{1,2}$ and the current study has late presentation as $>4$ weeks post-injury. A neglected fracture usually presents with distorted anatomy.

Due to the attachment of muscles, the fracture is more likely to displace. ${ }^{3}$ There were 27 (61.4\%) boys, and 17 (38.6\%) girls who were comparable to Shabir et al., ${ }^{4}$ who have $12(60 \%)$ boys, and $08(40 \%)$ girls in his study. Three children $(6.8 \%)$ had osteomyelitis (treated with curettage), seven (15.9\%) had pin-tract infections, which healed completely after removal of wires, while $34(77.3 \%)$ remained uneventful.

There was no nonunion in this study, which was comparable to Boz et al., ${ }^{2}$ who operated 69 cases of four-weeks older lateral condyle fractures and reported no case of nonunion. Good to excellent functional outcomes, using the Mayo elbow score, was achieved in $28(63.6 \%)$ and $12(27.2 \%)$ children, respectively (Mean \pm SD) $1.625 \pm 0.49187$. Similarly, Saraf et al. reported good to excellent results in his case series of five cases, ${ }^{1}$ and Mehmood et al. reported excellent results in $73.5 \%$ and good results in $13 \%$ children, ${ }^{5}$ which supported the findings of this study. Kumar et al. internally fixed three-week old neglected fracture lateral condyle with bone graft, and all fractures were united with $92 \%$ excellent, $05 \%$ good, and $03 \%$ poor results. ${ }^{3}$ Hung et al. ${ }^{6}$ study findings are contrary to our data due to different age group.

This study has limitations of small sample size and lack of randomisation of the population for a better level of evidence. Furthermore, the screw fixation can be used for the fixation of similarfractures.

\section{ETHICALAPPROVAL:}

The ethical approval was obtained from the Institutional Review Board of the Pakistan Society for the Rehabilitation of the Disabled on 28-06-2013.

\section{PATIENTS' CONSENT:}

Informed written consents were obtained from the children and their parents, where required.

\section{CONFLICT OF INTEREST:}

The authors have no conflict of interest to declare.

\section{AUTHOR'S CONTRIBUTION:}

$\mathrm{AH}$ : Design of the idea, critically revised the manuscript and final approval.

TS: Data collection, interpretation, and helped in writing the manuscript.

SFSG: Data analysis, interpretation, and authored the manuscript.

\section{REFERENCES}

1. Saraf SK, Khare GN. Late presentation of fractures of the lateral condyle of the humerus in children. Indian J Orthop 2011; 45(1):39-44. doi: 10.4103/0019-5413.67119.

2. Unal $B$, Ulusal $A E$, Vuruskaner $H$, Aydinoglu Y. Functional results of displaced lateral condyle fractures of the humerus with four-week K-wire fixation in children. Acta Orthop Traumatol Turc 2005; 39(3):193-8. 
3. Kumar N, Mehtani A, Yadav C, Raj R, Meena S, Barwar N. Delayed presentation of fracture of lateral condyle of humerus in pediatric age group treated by ORIF and ulnar peg grafting: A case series. J Orthop Allied Sci 2015; 3(1):12-6. DOI: 10.4103/2319-2585.155911

4. Shabir A, Tahir A, Sharief A, Imtiyaz H, Shahid H, Reyaz A. Delayed operative management of fractures of the lateral condyle of the humerus in children. Malays Orthop J 2015;
9(1):18-22. doi:10.5704/MOJ.1503.010.

5. Mahmood K, Shah FA, Atiq G, Shahab-ud-din, Mehsud WM, Qureshi AR, et al. Outcome of open reduction and internal fixation of fracture lateral condyle of humerus in children presented late. Pak J Surg 2014; 30(3):263-7.

6. Hung NN. Kirschner wire fixation of neglected lateral condylar fracture of the humerus in children. OA Lib J 2017; 4(1):2-20. doi: 10.4236/oalib.1103330. 